\title{
Adapting (to) Agile Methods: Exploring the Interplay of Agile Methods and Organizational Features
}

\author{
Christoph Fuchs \\ LMU Munich \\ fuchs@bwl.lmu.de
}

\begin{abstract}
It is a common understanding that agile methods are not implemented "one-to-one" from guidelines but are tailored to the specific conditions of organizations. This perspective, however, can be extended by taking into account that agile methods also have a considerable impact on organizational features of introducing firms. Against the backdrop of current application scenarios of agile methods in practice, this paper aims to capture and explain the interplay of agile methods and organizational features as well as their respective adaptations. By utilizing adaptive structuration theory as a theoretical research lens, I employ a qualitative-empirical research approach comprising four case studies. I find that the interplay of agile methods and organizational features represents a process of mutual adaptation that constitutes the organizational change in terms of agile methods' implementation. I further conclude that agile methods represent a vehicle to foster desired organizational change.
\end{abstract}

\section{Introduction}

Agile methods such as Scrum and eXtreme Programming (XP) emerged as practitioners' answer to traditional, plan-based approaches (e.g., waterfall model) to software development (SD) [1, 13]. Today, agile methods are a prevalent choice for information technology (IT) and SD projects and have thereby also spread beyond the IT and software industry [1, 27, 33]. Additionally, a current trend in practice is extending the usage of agile methods beyond the original application field of SD to novel contexts such as marketing projects and human resources (HR) [10, 27].

However, the implementation of agile methods entails crucial challenges for firms including unsuitable organizational structures and cultural values as well as inappropriate technical prerequisites $[5,17,21]$. As a result, agile methods are mostly not adopted "one-to- one" from guidelines, but are customized to firms' features [6, 9, 18]. Most empirical studies on the adaptation of agile methods emphasize their tailoring on the basis of practices according to organizational requirements [e.g., 14]. Nonetheless, agile methods can also have an impact on firms besides the changes in work processes and project outcomes (e.g., better product quality, higher customer satisfaction) [8]. Cao et al. [7], for instance, show that the implementation of agile methods results in the adaptation of IT funding processes to provide a better fit for agile IT projects. Tripp et al. [32] show that agile practices have a direct positive effect on the perceived job satisfaction of agile team members. Therefore, it is intriguing to explore how the implementation of agile methods can also impact organizational features such as organizational structures, teams' leadership styles, and cultural values.

In conclusion, I aim to build on the rich empirical foundation of the study of agile methods' adaptation and to extend the literature by taking a holistic perspective in order to capture adaptations of agile methods as well as organizational features. Therefore, I derive as underlying research questions (RQs):

RQ1: How are agile methods embedded in and adapted to organizational features?

RQ2: How does the implementation of agile methods shape organizational features?

While RQ1 follows the research tradition in the field of agile methods' adaptation, RQ2 expresses my extended, holistic view on the topic. By combining the insights from both RQs, I am able to explain the interplay of agile methods and organizational features as well as the related organizational change in terms of the implementation of agile methods.

To answer the RQs, I take into account that "practice is often ahead of research, and thus much can be learned from examining good practice" [14, p. 198]. Therefore, I study application scenarios of agile methods that represent current practice and are situated beyond pure SD or IT projects. Additionally, my holistic view on the interplay of agile methods and organizational features considers a processual lens. This enables me to gain an in-depth understanding of 
"underlying organizational change processes" [25, p. 4] such as adaptations of agile methods and organizational features. As a result, I adopt adaptive structuration theory (AST) as a theoretical research lens. I employ an explorative, qualitative-empirical approach by conducting multiple case studies. This study contributes to the understanding of the adaptation of agile methods and offers initial insights into the interplay of agile methods and organizational features.

\section{Theoretical background}

\subsection{Adaptation of agile methods}

The adaptation of agile methods is "the process of changing agile methods to align them with the needs of different projects and organizational environments" [8, p. 333-334]. Agile methods are customized by adopting, omitting or adapting agile practices (e.g., pair programming, daily standups) [8,9]. Whereas such a flexible approach to applying agile methods was seen critically in the past, today "agile practices [are viewed] as a "toolkit" to be applied as needed in a variety of project environments" [31, p. 4800].

Building on this, Fitzgerald et al. [14] investigate the feasibility of integrating agile practices from different methods (i.e., Scrum and XP). The authors empirically show that "an a la carte selection and tailoring of practices can work very well" [14, p. 197] and combining agile practices from different methods can be beneficial as they complement each other. Cao et al. [8] aim to provide a better understanding of the adaptation process. By means of AST and a qualitative-empirical research approach, the authors conclude that "agile practices are adapted and appropriated based on the project, organizational, and development context" [8, p. 342]. Tripp and Armstrong [31, p. 4799] further elaborate on these "forces that impact the manner in which agile methods are tailored" by examining organizational adoption motives and their relationship with the adaptation of agile methods. The researchers identify three overarching motives for adoption: to improve a) software quality, b) efficiency, and c) effectiveness. By matching these motives with the observed agile practices, the authors conclude that each motive is addressed by an individual constellation of agile practices [31].

Overall, research on the adaptation of agile methods offers rich empirical evidence and pertinent insights for practice. However, owing to the common focus on agile methods' adaptation, organizational features (e.g., structures, cultural values) are only referred to as factors that shape the individual firm's approach to customizing agile methods. This view can be extended by including organizational features not only as a shaping force but also as factors that are shaped by implementing agile practices. Academic and practical literature support this assumption. For instance, Cao et al. [7, p. 191] show that IT funding processes are adjusted to "accommodate the unique characteristics of agile IT projects". Denning [10] further suggests that the values and principles of agile methods (e.g., openness towards change, focus on constant communication, self-organization of teams) entailing the introduction of agile practices can also impact management styles, organizational structures, and cultural values. Consequently, I aim to apply a holistic, processual lens that captures the adaptation of agile methods and organizational features as well as enables me to comprehend their interplay.

\subsection{Adaptive structuration theory}

I select AST as theoretical research lens since AST has already been successfully employed in the context of agile methods' adaptation [e.g., 7, 8]. In addition, AST facilitates a holistic view on the interplay of agile methods and organizational features because AST considers contextual aspects (e.g., introduced agile practices, task environment) that influence the implementation of agile methods and the outcomes (i.e., adaptations) of human interaction with these aspects (i.e., teams applying adapted agile practices in an adapted organizational context) [11]. Finally, AST enables me to take a processual perspective on the adaptation of agile methods and organizational features, thus fostering a deeper understanding of the interplay of agile methods and organizational features and of the related organizational change $[11,25]$.

DeSanctis and Poole [11, p. 121] conceptualized AST on the basis of two concepts from social sciences, i.e., structuration and appropriation, to study "the role of advanced information technologies [AITs] in organization change". Referencing structuration theory [16], AST builds on social structures as core components that refer to rules and resources provided by characteristics of AITs and organizational features of the application context. The process of bringing these structures into action is referred to as structuration. The subsequent apparent "application of [such social] structures in a particular context" [8, p. $334]$ is considered as appropriation [11, 22]. Two types of structures result: structures embedded in AITs and organizational features, and structures embedded in action. These two types of structures are connected and mutually shape each other resulting in adaptations of AITs and organizations. With an ongoing reproduction of the two structure types, new social structures emerge and become institutionalized, constituting the 
manifested adaptations of firms and AITs as well as the related organizational change $[8,11]$.

Based on AST [11] and the tailored AST models of Cao and colleagues [7, 8], I derive an adapted AST model structured in three stages: input, process, and output (see Figure 1). I follow the argument that structure may not only be "provided by [technology, but] can also be provided by processes [i.e., agile methods] and organizations [i.e., organizational features]" [8, p. 334]. I take a holistic perspective and examine adaptations of the introduced agile methods and of the related organizational features.

The input stage encompasses the factors that provide social structures, namely agile methods and organizational features. First, the structure of agile methods is defined by the structural features (i.e., rules and principles) and the spirit (i.e., values) of the implemented agile methods. Structural features include principles and practices such as an iterative development approach, whereas an agile spirit entails, for instance, customer-centricity [2, 3]. Second, I consider structure provided by organizational features. Here, the organizational environment includes specific task characteristics (e.g., complexity) as well as aspects of hierarchies and cultural values of the agile methods' application field. Finally, the input element of the internal system of teams that are confronted with the implementation of agile methods includes, amongst others, team leadership style, team members' skills, and their experience with agile methods $[8,11]$.

In the process stage, the social structures of the input stage are brought into action (i.e., structuration) and are successively appropriated [11]. For instance, a firm's product development unit introduces the agile practice of daily standups to increase project transparency. Appropriation of structures is thereby characterized by four elements: appropriation moves, faithfulness of appropriation, instrumental use, and attitude towards appropriation. Appropriation moves are actions that illustrate how agile teams employ agile methods. The faithfulness of appropriation is the extent to which the structures in action fit the spirit of agile methods. The overall purpose of appropriation is considered in the element of instrumental use. Finally, attitude refers to "the perceptions and feelings of users toward the [use of agile methods]" [8, p. 334].

The appropriation of structures results in appropriated organizational and methodological outcomes, i.e., adaptations. Considering work processes-related outcomes, adaptations of agile methods are captured (e.g., changing daily standups to weekly standups). Adaptations of organizational features are evinced by the appropriated outcomes for the organizational environment (e.g., adaptation of organizational structures or task-specific aspects) and the team system-related adaptations (e.g., alteration of the leadership style in novice agile teams). Overall, I aim to portray these adaptations on an equal abstraction level taking into account elements such as practices and team roles $[8,11]$.

Additionally, initial outputs generated by the application of the input structures can "become additional sources of structure" [11, p. 128]. Such an emergent source of structure can, for instance, be constituted by an organization-wide spread of knowledge and interest in Scrum after an initial Scrum project has been successfully completed.

The output stage entails outcomes for the application context of agile methods with changes in performance measures, such as quality, costs, and time. Additionally, structures in action "are produced and reproduced" [11, p. 129] in social interaction (i.e., adaptation process) and are institutionalized over time, resulting in new social structures. These new structures represent reproductions of input or emergent structures or blendings of several of such structures. For this study, new structures depict the organizational change that is manifested as a result of all appropriated organizational and methodological outcomes (i.e., adaptations of agile methods and organizational features). Therefore, new structures portray the holistic results of the interplay of agile methods and organizational features (e.g., adapted organizational structure and work routine in a novice agile product development unit). Additionally, such new structures can, in turn, impact the adaptation process [11].

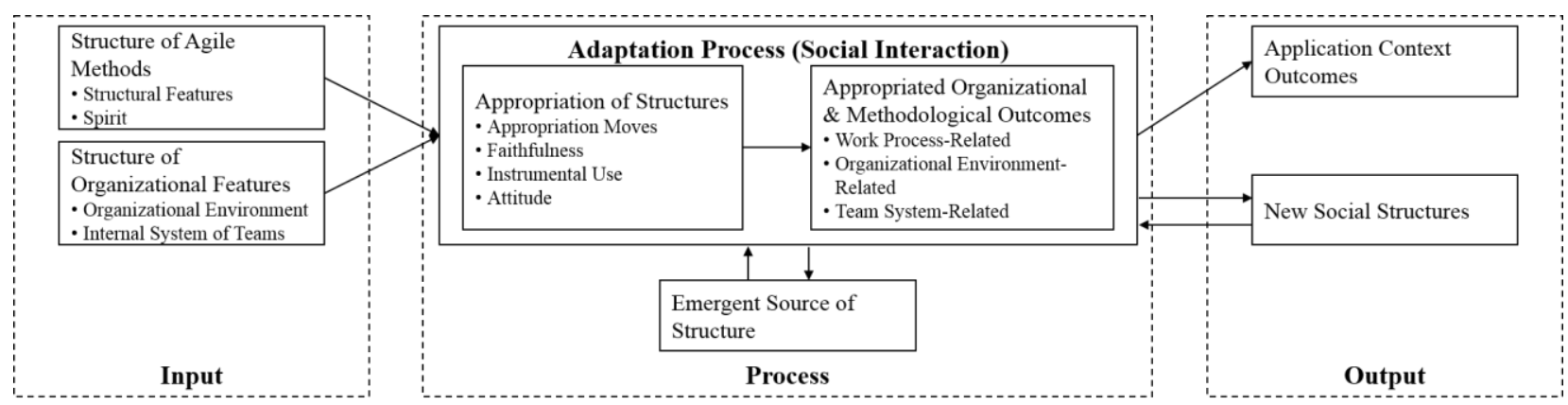

Figure 1. Adapted adaptive structuration theory model $[7,8,11]$ 
Table 1. Overview of sample and interviews

\begin{tabular}{|c|c|c|c|c|}
\hline & Case 1: DigBank & Case 2: DigAgent & Case 3: MechTech & Case 4: ElecTech \\
\hline Industry & Banking and finance & Digital agency & Mechanical engineering & Electronic engineering \\
\hline Revenue (2016) & $\sim 452$ billion $€$ & no information & $\sim 3$ billion $€$ & $\sim 339$ million $€$ \\
\hline Employees (2016) & $\sim 49,000$ & $\sim 250$ & $\sim 11,000$ & $\sim 2,000$ \\
\hline $\begin{array}{l}\text { Agile methods' } \\
\text { application scope / } \\
\text { related products }\end{array}$ & $\begin{array}{l}\text { Digitization center / } \\
\text { digitization concepts }\end{array}$ & $\begin{array}{l}\text { Consulting projects / } \\
\text { design concepts, micro } \\
\text { applications, websites, etc. }\end{array}$ & $\begin{array}{l}\text { Hardware and hardware- } \\
\text { related SD units / machine } \\
\text { tools and related software }\end{array}$ & $\begin{array}{l}\text { Digitization center / } \\
\text { concepts, hardware and } \\
\text { application software }\end{array}$ \\
\hline $\begin{array}{l}\text { Applied agile } \\
\text { methods }\end{array}$ & $\begin{array}{l}\text { Scrum, elemen } \\
\text { SAFe and LeS }\end{array}$ & $\begin{array}{l}\text { Scrum, Kanban, hybrid } \\
\text { approach }\end{array}$ & Scrum & $\begin{array}{l}\text { Scrum, Kanban, elements } \\
\text { of SAFe and LeSS }\end{array}$ \\
\hline $\begin{array}{l}\text { Number / dates of } \\
\text { interviews }\end{array}$ & $2 / 01 .-02.2018$ & $3 / 12.2017-02.2018$ & 5 / 06.2016, 03.2017, 02.2018 & $3 / 11.2017-01.2018$ \\
\hline $\begin{array}{l}\text { Position of } \\
\text { interviewees }\end{array}$ & \begin{tabular}{|l|}
- - Chief product \\
owner \\
- Agile team member \\
(Both in the \\
digitization center)
\end{tabular} & $\begin{array}{l}\text { - Organization developer } \\
\text { - Creative director: User } \\
\text { interface } \\
\text { - Senior project manager }\end{array}$ & $\begin{array}{l}\text { - Director machine tools } \\
\text { - Head of SD } \\
\text { - Head of machine-related SD } \\
\text { - Senior project manager } \\
\text { - Agile coach }\end{array}$ & $\begin{array}{l}\text { - Head of digitization } \\
\text { center } \\
\text { - Team leader } \\
\text { digitization center } \\
\text { - Senior project manager } \\
\end{array}$ \\
\hline
\end{tabular}

\section{Research method}

\subsection{Case study research}

The exploratory, multiple-case study research design is well suited to answer "how" and "why" questions and enables the examination of a real life problem in its natural context [36]. It also allows for a cross-case analysis of results and is less vulnerable to critique regarding the generalizability of results [36]. I adhere to guidelines of qualitative research to foster the rigor of my empirical study [12, 23, 36].

\subsection{Case selection and sample description}

I selected the cases purposefully [24] in two steps. First, I followed a criterion sampling logic to identify firms that recently (post 2013) implemented agile methods, which is in line with my approach to capture the current practice of agile methods. Second, I used a theoretical replication logic to select diverse cases with different application areas of agile methods to allow for contrasting findings [36]. The acquired sample includes four firms located in the same geographical region that differ in size, industry, application context of and experience with agile methods. I gathered data until I reached the point of no further novel information [36]. To ensure confidentially, I anonymized the cases (see Table 1 for an overview).

\subsection{Data collection and analysis}

The data collection took place June 2016 February 2018. During this time, I conducted interviews with firms' team members and managers that had extensive knowledge about the application of agile methods within their organization. All interviews were arranged via the author's professional network. The interviews were conducted on-site face-to-face or via telephone and lasted $45-90$ minutes. I used a semi-structured interview guide consisting of questions about a) the firms' current business situation, b) the firms' grasp of and experience with agile methods, c) the firms' implementation and usage history of agile methods, and d) the adaptations of agile methods and organizational features. With agile methods being a current trend in practice [27], I ensured to not observe approaches only labelled as "agile" but actual agile approaches by discussing the understanding of agile methods and their meaning for the firms with the interviewees. Selected interviews were carried out by two researchers of which one was a senior researcher. All interviews were recorded and transcribed verbatim. Besides performing data triangulation using secondary data (e.g., firm websites, management reports), I had partial access to internal data such as presentations about the strategy behind introducing agile methods. Case 1 includes fewer interviews since I had access to extensive internal documents. I used ATLAS.ti to build the case database and store, code, and analyze the qualitative data $[20,36]$. Because interviews at MechTech were completed earlier, I kept the case up to date with secondary data and catch-up interviews.

For the data analysis, I performed two cycles of coding. The first cycle of descriptive coding created an initial picture of the cases. This cycle was guided by the AST model and its aggregated categories such as structures embedded in agile methods and emergent sources of structure. In the second cycle, I revised the codes to address more specific AST aspects such as appropriation moves and depictions of agile spirit (see an exemplary coding scheme in Table 2). I devised 
case descriptions that I used to discuss the results with fellow researchers. Emerging differences were reviewed bilaterally and resolved consensually [20].

I present the case study results in two stages. First, I offer short descriptive case summaries and then discuss my findings with a cross-case analysis. In doing this, I provide findings for the organizational areas where agile methods are implemented. However, I also consider consequences for the entire firms such as changes in overall cultural values.

\section{Table 2. Illustration of coding scheme}

\begin{tabular}{|l|l|}
\hline \multicolumn{1}{|c|}{ Sample of interview statement } & \multicolumn{1}{|c|}{ Revised codes } \\
\hline $\begin{array}{l}\text { "We have bundled our digitization effort } \\
\text { in our [digitization center]. One essential } \\
\text { element here are agile methods." }\end{array}$ & $\begin{array}{l}\text { Organizational } \\
\text { environment } \\
\text { (Organizational } \\
\text { features) }\end{array}$ \\
\hline $\begin{array}{l}\text { "There were teams that loved Scrum at } \\
\text { first glance [...], and there were teams } \\
\text { that completely rejected agile methods, } \\
\text { because they lacked an agile mindset." }\end{array}$ & $\begin{array}{l}\text { Appropriation } \\
\text { moves / Attitude } \\
\text { (Appropriation) }\end{array}$ \\
\hline $\begin{array}{l}\text { "Issues are addressed more openly. Our } \\
\text { development is much faster. [...] The } \\
\text { transparency is incredible and helps us } \\
\text { with the commitment [of employees]." }\end{array}$ & $\begin{array}{l}\text { Time / } \\
\text { Transparency } \\
\text { (Application } \\
\text { context outcomes) }\end{array}$ \\
\hline
\end{tabular}

\section{Results}

\subsection{Case descriptions}

Case 1. DigBank is a longstanding large bank with a multi-national focus. To face the challenges of an increasingly digital world, DigBank founded a digitization center (DC) in late 2016 to aggregate all its digitization efforts. The DC is organized with respect to a scaled Scrum logic and its projects "are clustered according to three strategic directions: customerrelated operations, supporting topics [e.g., support processes] and the digitization of internal processes" (chief product owner). Each strategic direction includes up to nine different clusters that represent specific topics such as HR processes (i.e., digitization of internal processes). Individual agile projects are anchored within the thematic clusters. These projects develop, for instance, new talent management concepts (i.e., HR processes). The clusters include up to two chief product owners (CPOs), whereas the individual project teams are made up of a product owner, an interdisciplinary team and a Scrum Master. Agile coaches are available on the cluster level and are contact persons for the Scrum Masters. Employees of the core organization can apply for projects or are recommended by CPOs on the basis of a thematic project fit. The project team members return to their regular jobs after a predetermined period of one year. During their time as team members, they are fully committed to the project and exempt from their regular duties. CPOs are staffed with middle managers (e.g., team leaders) responsible for the respective digitization topic in the core organization, whereas Scrum Masters and agile coaches are mainly sourced externally. The DC currently spans twelve clusters with six to nine agile projects each, and team sizes of six to eight members. In total around 800 employees are involved.

Case 2. DigAgent, a digital agency which is part of an international advertising and marketing agency network. Starting in late 2016, the firm increasingly implemented Scrum for suitable consulting projects, considering products such as websites, micro applications, and design concepts. The implementation and spreading of agile methods within the firm was primarily driven bottom-up by interested employees, who tried to introduce "the agile mindset and convert it into actions to test whether it works small-scale [...] and if it works, [...] try to scale it" (organization developer). Now, agile methods are a default option for consulting projects. However, owing to resistance from clients and project teams, only about half of the projects are entirely executed with agile methods.

Case 3. MechTech is a medium-sized, familyowned engineering firm focusing on mechanical engineering and the development and distribution of machine tools. The organization has prior experience with agile methods since Scrum had been introduced in the machine-related SD unit years ago. However, only in 2014 did a new head of SD and the head of machine-related SD turn the former pseudo-agile processes into an actual agile approach. Currently, the machine-related SD unit routinely employs Scrum. About 150 employees work in teams of up to nine members on two essential software products related to specific machine tools. The interest in agile methods has grown organization-wide and spread into hardware development. As a result, the firm decided to develop "the most important [new] machine [according to Scrum]" (head of SD). This large machine development project included about 200 expert employees staffed from areas such as mechanical engineering and SD, resulting in a large-scale, interdisciplinary Scrum project in $2014-2016$.

Case 4. ElecTech is a family-owned, medium-sized manufacturer specializing in electromechanical systems. In late 2016, the firm founded a DC that is embedded directly below the executive board. The DC embraces five strategic fields: a) application SD, b) smart products, c) smart factory, d) smart operations, and e) data-driven-services. The DC includes about 25 people in six agile teams that carry out digitization projects according to Scrum and develop software, 
machine concepts, and digital solutions. The DC teams carry out their projects together with business units of the core firm aiming to spread the awareness for agile methods and "establish an agile community within the firm" (team leader DC). The project teams are crossfunctionally staffed according to the projects' contents and are mostly set up as actual Scrum teams.

\subsection{Cross-case analysis and discussion}

In this section, I discuss the cross-case analysis results based on the adapted AST model.

Input: All four firms initially implemented Scrum according to the guidelines [e.g., 30]. As the ElecTech team leader DC stated: "If we do projects according to Scrum, we have all the artifacts. All the planning meetings, the reviews and retros and all team roles". Therefore, the structural features of agile methods are similar in all four cases and refer to the tenets of Scrum including: sprint logic (i.e., iterative cycles), meetings (e.g., planning, daily standup, review, retrospectives), artifacts (e.g., product backlog, sprint backlog) and team roles (e.g., product owner, Scrum Master, development team). Considering the agile spirit, all firms embraced the "human-centric approach" (DigAgent, organization developer) of Scrum and further agile values such as, responsiveness to change, the rapid provision of working products, as well as open and continuous communication [2, 3, 30].

In terms of structures provided by organizational features, two approaches to the structural embedding of agile methods (i.e., organizational environment) were observed. DigBank and ElecTech decided to implement agile methods in a setting detached from the core firm, i.e., in their DCs. DigAgent and MechTech, however, chose to introduce Scrum directly in the respective areas, since both firms aimed to reach "true agility, fast dynamics, high adaptability [...] and the scaling of [agile] approaches" (MechTech, head of machine-related SD). Whereas the approach of ElecTech was intended to "spread the agile mindset" (ElecTech, senior project manager) within the firm, the agile approach at DigBank was not to be extended beyond the DC. Although the application scope of agile methods and the products differ across the firms (i.e., hardware development and SD at MechTech, SD at DigAgent and ElecTech, digitization concepts at DigBank), a common topic related to agile methods' introduction was the increasingly digital world and the associated digital transformation of firms. Such a digital transformation is interpreted as an IT-enabled business transformation where novel digital technologies are applied to innovate new business opportunities $[4,15]$. All interviewees agreed that the digital transformation represents a trigger for the implementation of agile methods since "agile is perfect when you work on something where you require fast results and rapid feedback to refine your ideas" (ElecTech, head of DC). Considering team internal systems, the firms contained almost no expertise in agile working routines and team leadership styles were command-and-control. Only the teams at MechTech had agile experience since Scrum had been introduced in the machine-related SD before, albeit unsuccessful.

Process: Overall, Scrum was appropriated well at all firms. This was reflected by various appropriation moves, for instance, at DigAgent, where "a running customer project was switched to Scrum" (DigAgent, senior project manager). Nonetheless, the adoption of agile methods was not completed in the cases, since even in the two DCs "there [are] still projects [...] where traditional methods [i.e., waterfall approach] are employed" (DigBank, CPO). The appropriation of agile methods' structures appeared faithful overall. For instance, at DigBank the DC teams were exempted from their regular line management responsibilities to focus on the work routine of Scrum. At MechTech, the management and team roles were transformed to fit Scrum, whereas team leaders became product owners and agile teams gained autonomy. The purpose of implementing agile methods was to gain agility within the processes as well as in cultural values (i.e., agile mindset). Nonetheless, agile practices and organizational features were adapted for instrumental use as well as faithful application (see next paragraph). Finally, the overall attitude toward agile methods was mainly positive in all the firms since the employees understood the benefits of certain agile features such as rapid iteration cycles and flexibility. Overall, this appropriation reflects structures in action that interact with the input structures resulting in appropriated organizational and methodological outcomes (i.e., adaptations) in relation to work processes, organizational environments and the teams involved.

In terms of work processes, all four firms initially implemented Scrum and tailored, as well as extended related agile practices. Sprint cycles varied depending on the products developed. At DigBank, for instance, sprints spanned one to two weeks since products did not necessarily include coding efforts but could "also be stakeholder workshops [on] devised HR concepts" (DigBank, agile team member). DigAgent included an independent design sprint that precedes the regular development sprint. Subsequent to this "sprint 0" the development sprints (two to three weeks) started and the design sprints continued, resulting in two parallel sprints. Owing to the dependence of machine-related SD teams on respective machines and their production rhythms of up to six weeks, a 12-week project phase was introduced at MechTech. During these 12 weeks, 
agile SD teams could autonomously decide on their sprint cycles (typically three weeks). Agile teams at ElecTech varied sprint length, engaging in "two to three week sprints depending on the topic and status of the projects" (ElecTech, team leader DC). All four firms reported implementing common Scrum meetings such as sprint planning, daily standups, sprint reviews and sprint retrospectives. Agile project teams at DigAgent added "refinement meetings in the middle of sprints to foster coordination [between designers and] developers" (DigAgent, organization developer) owing to the two parallel sprint cycles. All firms employed typical Scrum artifacts such as product and sprint backlogs and have agreed on a "definition of done". Physical Scrum boards were supported with digital collaboration tools (e.g., Jira) at all firms. The team roles of Scrum were also found in all firms, however, not always in their traditional function. In the project constellation at DigAgent, the client provided a product owner, whereas the agency allocated a project manager that should combine the roles of product owner and Scrum Master. In the case of ElecTech, "the allocation of roles [varied], although including every [Scrum] role [was] desired" (ElecTech, head of DC), owing to the constellation of interdisciplinary teams including members of the DC and of business units. In terms of the extension of agile approaches, DigBank and ElecTech both added elements of the Scaled Agile Framework (SAFe) [28] and Large-Scale Scrum (LeSS) [34] to address the scaled agile structures of their respective DCs. DigBank included the role of CPOs to manage the products of the thematic clusters. ElecTech built the DC "as one big area where multiple strategy fields and projects come together. As a result, there is one agile release train for each strategic field" (ElecTech, team leader DC) that projects are allocated to. DigAgent integrated further non-agile, but related concepts such as design thinking and atomic design into their consulting services. Finally, all firms adopted the practice of user stories and the idea of minimum viable products (originating from lean startup [26]) to describe shippable products and product increments.

In terms of the organizational environment, the agile approaches at DigBank and ElecTech were embedded in their DCs. In the case of DigAgent and MechTech, the implementation of agile methods directly impacted the organizational structures. At DigAgent, the existing four teams were divided into eight smaller teams in an attempt to "generate smaller units that could autonomously provide consulting services for clients" (DigAgent, creative director). In the case of MechTech, former team leaders in the machine-related SD unit were transferred to product owner roles within the agile teams but kept their disciplinary responsibilities. This resulted in agile team members being afraid to negotiate the scope of sprints since "the product owner was also responsible for the annual performance assessment" (MechTech, head of machine-related SD). To address this issue the new role of a "people manager" was introduced that pooled the disciplinary responsibilities in the unit. In terms of organizational cultures, different organizational worlds emerged, one with employees that embrace an agile mindset and another with employees lacking this mindset. In the case of the DCs, these two worlds were apparent. In the case of DigAgent "about one fourth [of the employees did] not want to be involved [in the agile approach]" (DigAgent, organization developer).

These new organizational structures and cultural values (i.e., agile mindset) also resulted in major adaptations of team systems. Since all four firms contained hierarchical leadership structures, the transition to new agile roles required "empathy and clear and respectful communication" (ElecTech, team leader DC). Members in leading positions often transitioned to roles within the agile teams (e.g., product owner) and thus mostly lost their spot as hierarchical superiors. However, this change was also problematic for novice development team members, since they were supposed to take more responsibility to emerge as self-organizing teams. Therefore, leadership and empowerment were two sides of the same coin. On the one hand, the former leading managers were required to adapt to their new roles as part of an agile Scrum team, often entailing a loss of power and a new leadership style (e.g., collaborative) [21]. On the other hand, agile team members had to embrace newly acquired autonomy. Across the four cases, a common picture emerged with many "motivated employees wanting to be part [of the agile initiatives] to shape the future of their firms" (DigBank, agile team member) and "others perceiving [the changes] as an unpleasant additional burden" (MechTech, agile coach). A related aspect are changes in stakeholder communication and collaboration, since stakeholders should be actively and continuously incorporated into agile projects $[2,3]$. The four firms selected different approaches to incorporate their stakeholders. At DigBank, relevant stakeholders such as the workers' council and specific business units (e.g., HR) were invited to participate in agile projects by sending members for the agile teams. At DigAgent, the clients were asked to provide a contact person to fulfill the role of the product owner. At MechTech and ElecTech, the product owners were responsible for the communication with stakeholders.

The appropriation of agile methods in the context of the organizational features resulted in emergent sources of structure. One common aspect was that the implementation of Scrum sparked firm-wide interest in agile methods. Therefore, further agile methods and 
practices were explored, also beyond the initial agile methods' application fields. At DigAgent, for instance, "the HR [unit] meanwhile organizes the recruitment process according to Kanban" (DigAgent, organization developer). To further elaborate their agile portfolio and offer an agile approach for projects where the conditions made the usage of Scrum less effective, the DC at ElecTech also added Kanban to their agile methods' portfolio. MechTech took a major step by launching the development of a crucial new machine tool together with the related software in an interdisciplinary Scrum project of 200 team members from various fields of expertise (e.g., SD, electrical engineering). The managers responsible at MechTech were aware from the start that "the project was not manageable with traditional project management, this was why [they] took the opportunity to initially apply Scrum in hardware development" (MechTech, head of machine-related SD). The firm rated the project a major success sparking organization-wide interest in agile methods. However, the growing interest in agile methods across all cases also led to the need to offer support for agile enthusiasts. Therefore, all four firms now offer workshops and trainings. DigAgent, for example, offers an "agile in a nutshell" course. The project management offices (PMOs) at MechTech and ElecTech acquired agile expertise by agile training programs themselves and offer support as agile coaches for interested teams.

Output: Overall, all interviewees reported that it is difficult to measure the success of implementing agile methods using cost and quality. However, the common opinion was that agile approaches led to faster project conclusions. Instead of hard facts, positive changes in soft aspects were reported. At DigAgent "the employees are much more satisfied because they know what they are working for" (DigAgent, creative director). This increase in transparency of work processes and outputs was evident in all four cases but turned out to be a two-edged sword. While, employees' and stakeholders' satisfaction increased, "transparency disclosed failures and problems and required a specific failure culture" (MechTech, senior project manager).

The interplay of adapted agile methods, adapted organizational features, and emergent sources of structure also manifested in new social structures in the firms. The two DCs elaborated their agile approaches and DigBank institutionalized a specific training program to educate Scrum Masters, since this role was mostly filled by external agile coaches. At ElecTech, the DC, together with the PMO, developed a portfolio of agile methods including Scrum and Kanban. With respect to the projects' conditions, a suitable agile approach can thus be compiled by means of the portfolio. DigAgent institutionalized a hybrid project management approach that resembles WaterScrum-Fall [35]. The approach embraces an iterative flow, close collaboration with, and high transparency towards the clients. However, the approach also covers upfront planning in the form of upstream sprints for concept design and scheduling. MechTech developed agile structures in its machine-related SD, institutionalizing a solution for leadership issues by distinguishing product and people management. Under the head of machine-related SD, two parallel structures emerged. One considers the product management including roles of CPOs and product owners, whereas the second structure accounts for people management comprising roles of people managers, agile coaches and Scrum Masters. People managers pool the disciplinary responsibilities for the team members, whereas agile coaches and Scrum Masters act as coaches. CPOs and product owners provide vision for projects which are staffed according to the required expertise. These new structures can again influence the adaptation process within the firms in the future.

\section{Conclusion}

\subsection{Theoretical implications}

With this paper I contribute to existing literature in three ways. First, I go beyond the previous applications of AST in the context of agile methods [e.g., 7, 8], which focused on appropriated outcomes of the adaptation process, and extend AST application in the tradition of agile methods' adaptation by providing empirical results for the entire AST model. With this, I am able to show that a complete consideration of AST is beneficial because I found, for instance, that the emergent sources of structure impact the adaptation process and shape the institutionalization of new social structures. This is evident in the example of ElecTech where a sophisticated portfolio of agile methods emerged owing to the need to handle the increased organization-wide interest in agile methods.

Secondly, given RQ1 - How are agile methods embedded in and adapted to organizational features? I conclude that different options to embed agile methods within organizations exist. Based on the analyzed data, the options can be roughly distinguished into separated (e.g., the DCs) and integrated approaches (e.g., MechTech). Independent from these options, I found that all firms tailored agile methods to their organizational requirements. This is evident in the case of DigAgent where a hybrid project management approach emerged to meet clients' needs for project control and upfront planning, but also in the case of ElecTech where the initially adopted Scrum framework 
is now part of an elaborated portfolio of agile methods including practices from Kanban.

Considering RQ2 - How does the implementation of agile methods shape organizational features? - I conclude that agile methods' implementation mostly addresses multiple features such as in the case of MechTech where the scaled Scrum approach resulted in the adaptation of hierarchies to form agile structures containing novel agile team roles (i.e., organizational environment). This structural transformation also led to changes in team systems in terms of leadership and team empowerment. Additionally, agile values spread and generated interest as is evident from the case of DigBank where it was never planned to extend agile methods beyond the DC, but the firm-wide interest led to the emergence of a specific training structure to educate internal Scrum Masters. With prior research focusing on the adaptation of agile methods [e.g., 8, 14], this study contributes to literature by showing that the implementation and tailoring of agile methods also results in adaptations of organizational features such as structures, cultural values, and teams' internal systems. As a result of the mutual adaptation of agile methods and organizational features, new structures emerge and institutionalize manifestations of the organizational change in the context of agile methods' usage. I summarize these findings in the following proposition:

Proposition 1: The interplay of agile methods and organizational features in terms of the implementation of agile methods represents a process of mutual adaptation that constitutes organizational change.

Finally, I derive from the findings that firms work towards specific effects of the implementation of agile methods to foster desired organizational change. For instance, across all cases interviewees reported that their firms explicitly wanted to introduce cultural values associated with agile methods and an agile mindset, such as transparency, openness and adaptability towards change, and a positive failure culture $[2,3,10]$. With literature mainly focusing on the suitability of organizational features (e.g., culture [e.g., 19, 29]) for the introduction of agile methods, such a view can be fruitful for future research. Consequently, I derive the following proposition:

Proposition 2: Firms utilize agile methods as a vehicle to foster desired organizational change.

\subsection{Practical implications}

Referring to practical implications, practitioners should be aware that transparency is a double-edged sword that can result in greater commitment of employees but can also foster resistance. In addition, not all employees desire more responsibility or autonomy. Therefore, practitioners should evaluate where the use of agile methods and related values are most expedient. Additionally, transparency about the agile approach and the underlying purpose of its implementation is crucial for its acceptance as this counteracts uncertainty among employees. As the usage of agile methods can spark increased interest in an agile way of working, firms should prepare for such instances. PMOs, as in the cases of MechTech and ElecTech, play a crucial factor in supporting agile enthusiasts. Consequently, gaining and sharing of agile expertise appears essential for a successful implementation and a potential spreading of agile methods and values. Finally, to capture the success of introducing agile methods, firms should develop initial measures beforehand. These can subsequently be customized as the process of mutual adaptation progresses. Also, measures for soft factors such as employee satisfaction should be included.

\section{Limitations and future research}

This paper is not without limitations. First, I studied firms in one geographical area with specific organizational features. Although, I aimed at heterogeneous cases, these firm-specific restrictions constrain the generalizability of the results, adding to the limited generalizability of the study's qualitative research approach. In addition, respondents may have presented the agile approaches in their firms in an overly positive light since their careers may depend on its success (e.g., managers). Finally, as the study takes a holistic view with a focus on the adaptation of agile methods and organizational features as well as their interplay, emerging topics such as the fit of agile methods to an increasingly digital world and an associated organizational digital transformation could not be discussed in-depth. However, this fit appears as a fruitful topic for future research.

Additionally, this study raised the question of an altered relationship between agile methods and organizational features, where agile methods are actively employed to alter, for instance, cultural values. Therefore, it appears promising to explore the impact of agile methods on different organizational features in-depth and test the offered propositions empirically.

\section{References}

[1] Abrahamsson, P., Conboy, K., and Wang, X., "'Lots Done, More to Do': The Current State of Agile Systems Development Research", European Journal of Information Systems, 18(4), 2009, pp. 281-284.

[2] Beck, K., Beedle, M., Van Bennekum, A., Cockburn, A., Cunningham, W., Fowler, M., Grenning, J., Highsmith, J., Hunt, A., Jeffries, R., Kern, J., Marick, B., Martin, R.C., 
Mellor, S., Schwaber, K., Sutherland, J., and Thomas, D., "Manifesto for Agile Software Development", 2001.

[3] Beck, K., Beedle, M., Van Bennekum, A., Cockburn, A., Cunningham, W., Fowler, M., Grenning, J., Highsmith, J., Hunt, A., Jeffries, R., Kern, J., Marick, B., Martin, R.C., Mellor, S., Schwaber, K., Sutherland, J., and Thomas, D., "Principles behind the Agile Manifesto", 2001.

[4] Besson, P., and Rowe, F., "Strategizing Information Systems-Enabled Organizational Transformation: A Transdisciplinary Review and New Directions", The Journal of Strategic Information Systems, 21(2), 2012, pp. 103-124.

[5] Boehm, B., and Turner, R., "Management Challenges to Implementing Agile Processes in Traditional Development Organizations", Project Management, 22(5), 2005, pp. 30-39.

[6] Campanelli, A.S., and Parreiras, F.S., "Agile Methods Tailoring - A Systematic Literature Review", The Journal of Systems and Software, 110(12), 2015, pp. 85-100.

[7] Cao, L., Mohan, K., Ramesh, B., and Sarkar, S., "Adapting Funding Processes for Agile IT Projects: An Empirical Investigation", European Journal of Information Systems, 22(2), 2013, pp. 191-205.

[8] Cao, L., Mohan, K., Xu, P., and Ramesh, B., "A Framework for Adapting Agile Development Methodologies", European Journal of Information Systems, 18(4), 2009, pp. 332-343.

[9] Conboy, K., and Fitzgerald, B., "Method and Developer Characteristics for Effective Agile Method Tailoring: A Study of XP Expert Opinion", ACM Transactions on Software Engineering and Methodology, 20(1), 2010, pp. 130.

[10] Denning, S., The Age of Agile - How Smart Companies Are Transforming the Way Work Gets Done, American Management Association, New York, USA, 2018.

[11] DeSanctis, G., and Poole, M.S., "Capturing the Complexity in Advanced Technology Use: Adaptive Structuration Theory", Organization Science, 5(2), 1994, pp. 121-147.

[12] Dubé, L., and Paré, G., "Rigor in Information Systems Positivist Case Research: Current Practices, Trends, and Recommendations", MIS Quarterly, 27(4), 2003, pp. 597636.

[13] Dyba, T., and Dingsøyr, T., "Empirical Studies of Agile Software Development: A Systematic Review", Information and Software Technology, 50(9-10), 2008, pp. 833-859.

[14] Fitzgerald, B., Hartnett, G., and Conboy, K., "Customising Agile Methods to Software Practices at Intel Shannon", European Journal of Information Systems, 15(2), 2006, pp. 197-210.

[15] Fitzgerald, M., Kruschwitz, N., Bonnet, D., and Welch, M., "Embracing Digital Technology - A New Strategic Imperative", Massachusetts Institute of Technology, Boston, USA, 2013 pp. 1-12.

[16] Giddens, A., Central Problems in Social Theory, University of California Press, Berkeley, 1979.

[17] Hekkala, R., Stein, M.-K., Rossi, M., and Smolander, K., "Challenges in Transitioning to an Agile Way of Working", Proceedings of the 50th Hawaii International Conference on System Sciences, 2017, pp. 5869-5878.

[18] Hron, M., and Obwegeser, N., "Scrum in Practice: An Overview of Scrum Adaptations", Proceedings of the 51st
Hawaii International Conference on System Sciences, 2018, pp. 5445-5454.

[19] Iivari, J., and Iivari, N., "The Relationship between Organizational Culture and the Deployment of Agile Methods", Information and Software Technology, 53(5), 2011, pp. 509-520.

[20] Miles, M.B., Huberman, A.M., and Saldana, J., Qualitative Data Analysis - A Methods Sourcebook, SAGE Publications, 3rd ed., Thousand Oaks, USA, 2013.

[21] Nerur, S., Mahapatra, R., and Mangalaraj, G., "Challenges of Migrating to Agile Methodologies", Communications of the ACM, 48(5), 2005, pp. 72-78.

[22] Ollman, B., Alienation: Marx's Conception of Man in Capitalist Society, Cambrige University Press, Cambridge, 1971.

[23] Paré, G., "Investigating Information Systems with Positivist Case Research", Communications of the Association for Information Systems, 13(1), 2004, pp. 233264.

[24] Patton, M.Q., Qualitative Research \& Evaluation SAGE Publications, 3rd ed., Thousand Oaks, USA, 2002.

[25] Poole, M.S., Van De Ven, A.H., Dooley, K., and Holmes, M.E., Organizational Change and Innovation Processes - Theory and Methods for Research, Oxford University Press, Oxford, UK, 2000.

[26] Ries, E., The Lean Startup - How Constant Innovation Creates Radically Successful Businesses, Portfolio Penguin, London, UK, 2011.

[27] Rigby, D.K., Sutherland, J., and Takeuchi, H., "Embracing Agile - How to Master the Process that's Transforming Management", Harvard Business Review, 94(5), 2016, pp. 41-50.

[28] Scaled Agile, "SAFe® 4.5 Introduction - Overview of the Scaled Agile Framework ${ }^{\circledR}$ for Lean Enterprises", Scaled Agile, Inc., Boulder, USA, 2017.

[29] Strode, D.E., Huff, S.L., and Tretiakov, A., "The Impact of Organizational Culture on Agile Method Use", Proceedings of the 42nd Hawaii International Conference on System Sciences, 2009, pp. 1-9.

[30] Sutherland, J., and Schwaber, K., "The Scrum Guide The Definitive Guide to Scrum: The Rules of the Game", ScrumInc., 2016.

[31] Tripp, J.F., and Armstrong, D.J., "Exploring the Relationship Between Organizational Adoption Motives and the Tailoring of Agile Methods", Proceedings of the 47th Hawaii International Conference on System Sciences, 2014, pp. 4799-4806.

[32] Tripp, J.F., Riemenschneider, C., and Thatcher, J.B., "Job Satisfaction in Agile Development Teams: Agile Development as Work Redesign", Journal of the Association for Information Systems, 17(4), 2016, pp. 267-307.

[33] Version One, "State of Agile Report, 11th Annual Survey", 2017, https://explore.versionone.com/state-ofagile/versionone-11th-annual-state-of-agile-report-2

[34] Vodde, B., and Larman, C., "LeSS Framework", The LeSS Company B.V., 2014.

[35] West, D., "Water-Scrum-Fall Is the Reality of Agile for Most Organizations Today", Forrester Research, Cambridge, USA, 2011

[36] Yin, R.K., Case Study Research - Design and Methods, SAGE Publications, 5th ed., Thousand Oaks, USA, 2013. 